\title{
Perception
}

\section{du « bon » médecin dentiste : enquête auprès des patients marocains}

\section{Perception of the "voucher" doctor dentist: survey with the Moroccan patients}

\section{Résumé}

\section{MOTS-CLEFS :}

- Perception, appréciation, dentiste, Maroc, patients

\section{KEYWORDS:}

- Perception, evaluation, dentist, Morocco, patients
Objectifs : Le but de notre enquête est d'étudier les perceptions des patients marocains du «bon » médecin dentiste et de déterminer l'image qu'ils ont aujourd'hui du médecin dentiste.

Matériel et méthode : L'enquête est effectuée chez un échantillon composé de 200 patients marocains, dans les deux villes Rabat et Salé, en utilisant un questionnaire anonyme à réponses ouvertes. Une étude statistique descriptive et analytique a été menée pour traiter les donnés.

Résultats : D'après les résultats de l'étude statistique, $84,6 \%$ des patients ont une bonne image du médecin dentiste.

Les résultats de notre enquête ont aussi révélé que la qualité de la relation humaine (comportement du médecin dentiste $69 \%$ et sa communication avec le patient $64 \%$ ) est le critère le plus déterminant du « bon » médecin dentiste, suivi du contexte du travail $68,8 \%$.

Viennent ensuite les honoraires qui représentent $23,5 \%$, ainsi que l'hygiène et la stérilisation avec $16 \%$.

Enfin les facteurs personnels rattachés au médecin dentiste et la présentation du cabinet occupent $6,5 \%$ de la perception du « bon » médecin dentiste.

Conclusion : Le comportement et la communication du médecin dentiste étant très importants dans son appréciation par les patients, il serait judicieux de leur prêter l'attention qu'ils méritent par les dentistes ainsi que lors des études dentaires.

\section{Abstract}

Goal: The purpose of the study is to determine the perception that it makes the Moroccans patient of a "good" dentist.

Material and method: The study concerned 200 Moroccan patients, in both cities Rabat and Salé. By using an anonymous questionnaire, with opened answers. A descriptive and analytical statistical study was led to treat results.

Results: According to the results of statistical study, $84.6 \%$ of Moroccans patient have a good picture of the doctor dentist.

The results revealed that the quality of human relation (behavior of the doctor $69 \%$ and his communication with the patient $64 \%$ ) is the most decisive criteria of the "good" dentist, followed by the context of job $68.8 \%$.

The fee comes then which represent $23.5 \%$, as well as hygiene and sterilization with $16 \%$.

Finally the personality of a dentist and the presentation of the office présent 6.5\% the perception of a "good" dentist.

Conclusion: Behavior and communication of the dentist are very important in her evaluation by the patients, it would be judicious to pay them the attention which they deserve by the dentists as well as during dental studies. 


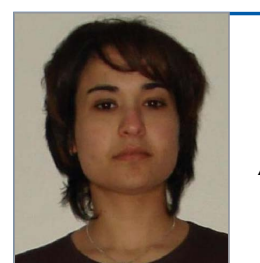

Jihane SLAOUI HASNAOUI, Professeur assistant au Service de Prothèse Adjointe - Centre de traitement et de consultation dentaire de RABAT

Asmâa BENDELHA, Chirurgien-dentiste - secteur libéral

EI Miloud RAHMANI, Professeur de l'Enseignement Supérieur au département de prothèse adjointe - Centre de traitement et de consultation dentaire de RABAT

Sanâa CHALA, Professeur Agrégé au département d'odontologie conservatrice, Centre de traitement et de consultation dentaire de RABAT

Faiza BENFDIL, Professeur Agrégé au département de prothèse adjointe - Faculté de médecine dentaire de RABAT - Centre de traitement et de consultation dentaire de RABAT

\section{INTRODUCTION}

"Qu'est-ce qu'un bon dentiste? Comment pouvez-vous en trouver. Une réponse à ces questions vous permettra d'obtenir le meilleur traitement dentaire possible».

Ce sont ces questions le plus souvent posées par les patients, à la recherche d'un professionnel de santé auquel ils souhaitent s'adresser.

L'expression «bon» médecin dentiste est souvent utilisée par les patients pour désigner le médecin auquel ils se réfèrent et chez qui leurs attentes étaient satisfaites.

Pourquoi ? Tout simplement parce que, dans les rapports humains, perception = réalité. La perception que se font les patients est leur "réalité ». Vous pouvez être le meilleur praticien du monde, si le patient ne le perçoit pas, il vous considérera comme les autres. Votre réputation se fait sur la valeur «perçue » de la qualité de vos traitements. Or, nous savons tous que le patient n'a ni les compétences ni les aptitudes pour juger la qualité réelle des traitements. Il nous juge donc sur les seuls éléments susceptibles d'entrer dans son champ de perception [1].

Le dentiste est parmi les personnages les plus représentés dans les histoires comiques ou terrifiantes, à travers les stéréotypes du patient victime et du dentiste bourreau.

Nous connaissons les innombrables représentations artistiques, cinématographiques ou même publicitaires sur le sujet.

La terreur est toujours au rendez-vous! Néanmoins, le stéréotype du dentiste puissant, craint, « l'arracheur de dents », sadique, qui vit dans l'inconscient collectif, semble en voie d'extinction. Il est remplacé par le spécialiste à qui on reconnaît une grande compétence technique, qui restaure la fonction, la puissance masticatoire et l'esthétique de la bouche.

Comme le dit encore Marie Bonaparte, le dentiste contemporain peut être comparé à la bonne "mère nature ». Elle console, non seulement, l'enfant « de la douleur de la castration symbolique ", de l'humiliation de la dent de lait tombée ou arrachée, en la remplaçant, mais en la remplaçant par une dent " d'une plus grande dimension, d'une plus grande force, d'une plus grande dignité, par une dent d'adulte ».

Et comme la «mère nature », le dentiste d'aujourd'hui revalorise donc l'image de soi aussi bien personnelle que relationnelle. Il appartient à présent à la médecine de la qualité de vie.

Cette fonction explique le phénomène appelé quelquefois « transfert », mais aussi « attachement, admiration, dépendance, confiance, amour...» vécu par nos patients, qu'ils soient " oisif, mondain, ingénieur, avocat ou même médecin », et qui rejettent en bloc les dentistes pour aimer « leur dentiste».

On choisit, alors « son » dentiste, à la fois spécialiste et médecin de famille, ce qui explique toute son importance.

L'enseignement de la chirurgie dentaire est constitué surtout de l'acquis d'un savoir fondamental et trop rarement de l'apprentissage de la relation de soins. Comme l'art est constitué d'un don inné et d'un acquis qu'est l'apprentissage et le travail, de même la relation s'améliore, s'apprend, se travaille.

Dans le cadre d'une analyse de la notion du «bon " médecin dentiste, nous avons mené une enquête auprès de 200 patients. L'objectif principal de ce travail est d'étudier les représentations que se font les patients marocains du «bon» médecin dentiste et secondairement l'enquête vise à déterminer la perception du dentiste par les marocains et de déterminer les facteurs associés au choix du médecin dentiste par les patients marocains.

\section{MATÉRIEL ET MÉTHODE}

Il s'agit d'une enquête transversale, menée dans deux villes du royaume, Salé et Rabat, du 02/06/11 jusqu'au 30/08/11. Elle s'est basée sur le recueil des points de vue des patients concernant le médecin dentiste et le « bon » médecin dentiste, à la base d'un questionnaire. 
Notre population était constituée d'un échantillon de 200 individus volontaires de nationalité marocaine, âgés de 18 ans et plus. Dans le but d'obtenir un échantillon homogène, nous avons choisi de mener l'enquête dans des endroits susceptibles d'accueillir toutes les tranches d'individus représentatives de la population mère.

Le questionnaire a d'abord été validé par une pré-enquête, afin de déceler les erreurs et les imperfections de celui-ci. Il a ensuite été distribué dans les cabinets dentaires, les administrations, et les lieux publics sans aucune sélection.

Les données ont été recueillies directement auprès des patients en expliquant au préalable le but de l'enquête. Ces personnes ont rempli individuellement le questionnaire et nous ne sommes intervenus qu'en cas de traduction, de non-compréhension ou de non réponse à certaines questions, pour obtenir le maximum de renseignements, sans toutefois les influencer. Concernant les patients analphabètes, nous sommes intervenus aussi pour la rédaction de leurs réponses.

Le questionnaire était un imprimé anonyme constitué d'une page. Il comportait :

I une fiche d'identification de l'individu dont les réponses étaient fermées (réponses prédéterminées) portant des indications sur l'âge, le sexe, la profession, le lieu de résidence, le niveau d'étude, le motif de consultation, le revenu mensuel de la famille et la consultation antérieure chez le médecin dentiste

12 questions à réponses ouvertes portant sur la perception de la population du médecin dentiste :

- Pour vous, qu'est ce qu'un médecin dentiste?

- Pour vous, qu'est ce qu'un « bon » médecin dentiste?

Pour les réponses ouvertes, les verbatim de toutes les réponses remises ont été systématiquement analysées. Nous avons colligé les mots clefs correspondant à un verbe, un adjectif ou une locution employés par les répondants et concernant une qualité, une caractéristique ou un attribut potentiellement rattachables au médecin dentiste.

Ensuite nous les avons classé par catégorie, et une liste a été établie pour le médecin dentiste et le «bon » médecin dentiste (Tableau I).

La saisie des données a été réalisée avec le logiciel Excel 2007, l'analyse des résultats a été effectuée avec le logiciel Statistique Package for Social Science (SPSS version 13.0) pour Windows. Les variables recueillies ont été exprimées en effectif et pourcentage et Le test khi carré (khi2) a permis la comparaison des distributions observées entre deux variables qualitatives.

\section{RÉSULTAT}

$71 \%$ de l'échantillon sont de la région Rabat-SaléZemmour-Zair, les autres sont d'origines diverses: Errachidia, Meknès, Azrou, Khénifra, Marrakech, Casablanca, Agadir, Tanger, Fès, Berkane, Bouarfa, Tétouan.

$64 \%$ des participants appartiennent à la tranche d'âge entre 18-39 ans.

$57 \%$ de la population enquêtée sont des femmes.

$40 \%$ ont une activité professionnelle régulière, et $25 \%$ sont des étudiants. $91,5 \%$ des patients habitent en milieu urbain. $69 \%$ des patients ont un niveau universitaire.

$85 \%$ des patients ayant participé à notre enquête, déclarent avoir consulté un médecin dentiste. Concernant le motif de consultation, $34 \%$ des patients consultaient pour motif de douleur, $24 \%$ pour souci esthétique, et $21 \%$ des patients pour un problème fonctionnel.

Les participants à notre enquête ont un revenu mensuel moyen : $40 \%$ inférieur à 4000 dirhams, $33 \%$ des patients entre 4000 et 8000 dirhams, $26 \%$ entre 8000 et 30000 dirhams et $1 \%$ supérieur à 30000 dirhams.

$68 \%$ des patients considèrent le médecin dentiste comme un soignant, $19 \%$ des patients le voient comme un professionnel de santé et $7 \%$ patients le considèrent comme un arracheur de dents (Fig. 1).

Pour les 200 participants à l'enquête, les facteurs qui rentrent dans leur perception du «bon » médecin dentiste sont répartis comme suit : (Fig. 2)

$169 \%$ des patients exposaient la compétence,

1 $68,8 \%$ des patients indiquaient le comportement du médecin dentiste,

$164 \%$ des patients exprimaient la communication avec le médecin dentiste,

\section{DISCUSSION}

Pendant la phase de la réalisation de l'enquête, nous avons rencontré plusieurs difficultés :

\ 40 patients ont refusé le remplissage du questionnaire. Ce refus vient dans la majorité des cas avant même qu'on présente le sujet ou le but de l'enquête et malgré l'anonymat du questionnaire ; dès lors, il est impossible de prévenir l'influence que cela a pu avoir sur la nature des résultats obtenus.

$\checkmark$ les personnes ont en outre été interrogées par écrit, ce qui induit un biais social très discriminant. Le niveau d'instruction des individus de l'échantillon était parfois faible, ce qui rend difficile l'entente entre l'enquêteur et la personne questionnée. 
$\checkmark$ Tableau I : Les facteurs intervenant dans l'appréciation du médecin dentiste par les patients.

\section{Liste du médecin dentiste}

\begin{tabular}{|c|c|}
\hline Soignant & $\begin{array}{l}\text { - fait un traitement dentaire } \\
\text { - soigne les dents } \\
\text { - fait des appareils dentaires pour bien } \\
\text { mastiquer, et remplaçant les dents } \\
\text { détruites } \\
\text { - soulage la douleur } \\
\text { - aide et secours } \\
\text { - soignant des dents et gencive } \\
\text { - soigne les caries } \\
\text { - soigne la bouche } \\
\text { - homme ou femme qui répare les dents } \\
\text { - trouve solution à tous les problèmes } \\
\text { dentaires }\end{array}$ \\
\hline Artiste & $\begin{array}{l}\text { - redonne le beau sourire } \\
\text { - redonne sourire aux gens } \\
\text { - améliore le sourire et l'état des dents } \\
\text { mal alignés } \\
\text { - aide les gens à avoir le beau sourire } \\
\text { et le bon look } \\
\text { - donne accès à une belle dentition, } \\
\text { véritable artiste des dents } \\
\text { - améliore l'apparence des dents } \\
\text { - la médecine dentaire est un art, et non } \\
\text { une science exacte } \\
\text { - rend les dents plus belles }\end{array}$ \\
\hline $\begin{array}{l}\text { Arracheur } \\
\text { de dents }\end{array}$ & $\begin{array}{l}\text { - mécanicien des dents } \\
\text { - arracheur des dents } \\
\text { - personne qui arrache les dents }\end{array}$ \\
\hline $\begin{array}{l}\text { Professionnel } \\
\text { de la santé }\end{array}$ & $\begin{array}{l}\text { - personnel de la santé } \\
\text { - médecin dentiste } \\
\text { - spécialiste des dents } \\
\text { - praticien diplômé } \\
\text { - pratique légalement la médecine } \\
\text { dentaire } \\
\text { - médecin comme tous les autres } \\
\text { médecins } \\
\text { - métier noble } \\
\text { - il a un cabinet dentaire } \\
\text { - professionnel de la santé qui s'occupe } \\
\text { des dents } \\
\text { - spécialisé en chirurgie dentaire } \\
\text { - spécialiste dans le domaine de } \\
\text { la dentisterie } \\
\text { - médecin dentiste } \neq \text { charlatan }\end{array}$ \\
\hline $\begin{array}{l}\text { Bonne } \\
\text { réputation }\end{array}$ & - qui a une bonne réputation \\
\hline
\end{tabular}

\section{Liste du " bon » médecin dentiste}

\begin{tabular}{|c|c|}
\hline $\begin{array}{l}\text { Compétence } \\
\text { et méthode } \\
\text { de travail }\end{array}$ & $\begin{array}{l}\text { - Compétence technique } \\
\text { - Efficace } \\
\text { - Techniques récentes } \\
\text { - Sans douleurs } \\
\text { - Travaille avec douceur } \\
\text { - Rapide dans l'exécution } \\
\text { - soins de qualité } \\
\text { - travail en équipe } \\
\text { - expérimenté }\end{array}$ \\
\hline Comportement & $\begin{array}{l}\text { - sympathique } \\
\text { - modeste } \\
\text { - sérieux } \\
\text { - souriant } \\
\text { - courtoisie et respect du patient } \\
\text { - respect des rendez-vous } \\
\text { - accueillant } \\
\text { - crée un bon climat, met à l'aise } \\
\text { - attentionnée } \\
\text { - gentil } \\
\text { - honnête }\end{array}$ \\
\hline Communication & $\begin{array}{l}\text { - communique avec les patients } \\
\text { - explique ce qui est pratique et } \\
\text { les procédures du traitement } \\
\text { - écoute } \\
\text { - empathie } \\
\text { - rassure } \\
\text { - adapte les explications selon } \\
\text { le niveau de compréhension } \\
\text { du patient } \\
\text { - procure assez de temps aux patients } \\
\text { - digne de confiance } \\
\text { - clair lors des explications }\end{array}$ \\
\hline F.P.M.D & $\begin{array}{l}\text { - sexe féminin } \\
\text { - âgée } \\
\text { - avoir la conscience professionnelle }\end{array}$ \\
\hline Honoraire & $\begin{array}{l}\text { - pas cher } \\
\text { - fait des facilités de paiement } \\
\text { - paiement sur tranches, différée } \\
\text { - prix adapté au cas social du patient }\end{array}$ \\
\hline $\begin{array}{l}\text { Présentation } \\
\text { et situation } \\
\text { du cabinet }\end{array}$ & $\begin{array}{l}\text { - cabinet spacieux avec } \\
\text { divertissement intéressant } \\
\text { - fauteuil confortable } \\
\text { - proche du domicile, lieu du travail } \\
\text { - d'accès facile } \\
\text { - équipements de dernière } \\
\text { technologie }\end{array}$ \\
\hline H et $S$ & $\begin{array}{l}\text { - propreté et asepsie } \\
\text { - hygiène rigoureuse }\end{array}$ \\
\hline Autres & $\begin{array}{l}\text { - le bon dentiste n'existe pas } \\
\text { - Celui qui s'adapte à mes horaires } \\
\text { de travail } \\
\text { - joignable en week end } \\
\text { - son travail doit être accompli } \\
\text { en trois séances } \\
\text { - parle peu }\end{array}$ \\
\hline
\end{tabular}

* F.P.M.D : facteurs liés à la personne du médecin dentiste.

${ }^{*} \boldsymbol{H}$ et $S$ : Hygiène et stérilisation. 


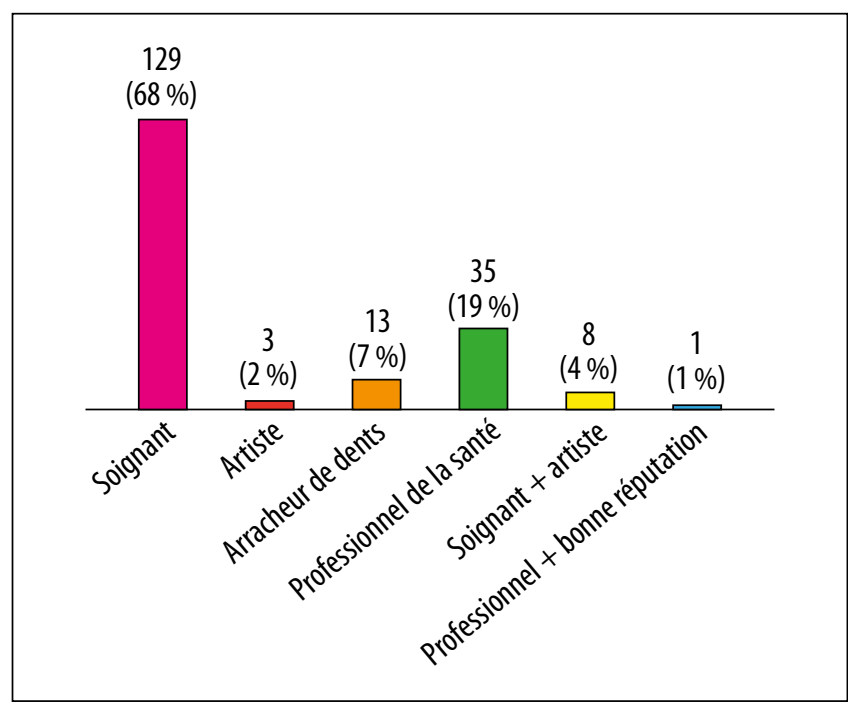

$\triangle$ Fig. I : Répartition de l'échantillon selon la perception du médecin dentiste.

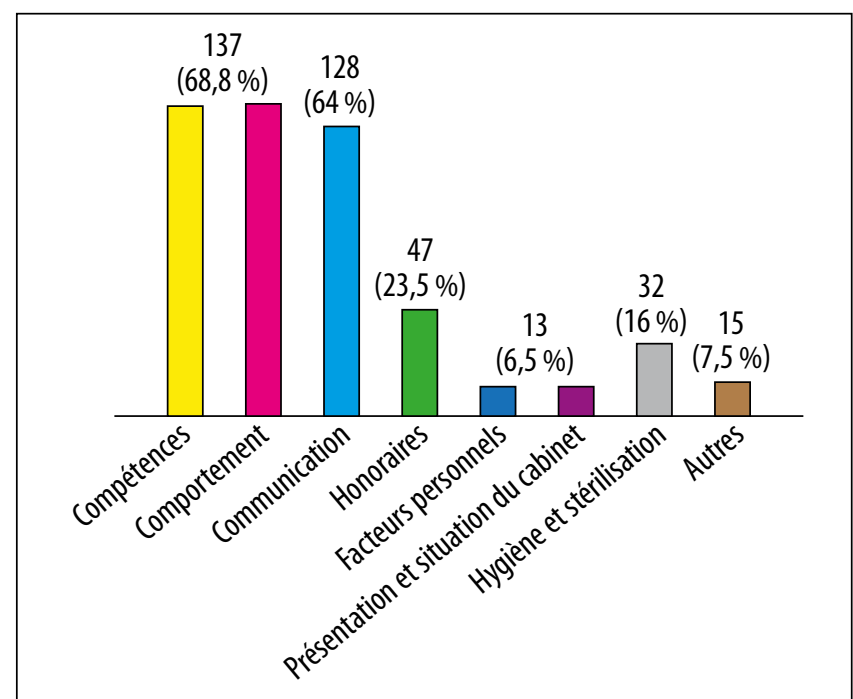

$\triangle$ Fig. II : Répartition de l'échantillon selon la perception du " bon " médecin dentiste.

\section{PERCEPTION DU MÉDECIN DENTISTE}

D'après une enquête réalisée en 2007 [2] par l'association dentaire française mettant en évidence la perception des chirurgiens dentistes et de la santé dentaire par les français, $95 \%$ des français déclarent avoir une bonne image du métier du chirurgien dentiste et $41 \%$ une très bonne image du métier. Cette bonne image est plus forte dès lors que les personnes fréquentent d'avantage les cabinets dentaires.

Ceci vient appuyer les résultats de notre enquête. Les patients marocains ont une bonne image du médecin dentiste puisque $84,6 \%$ d'entre eux le reconnaissent comme : soignant, artiste, professionnel, et celui qui a une bonne réputation. Heureusement seuls $7 \%$ continuent de le considérer jusqu'à aujourd'hui comme un arracheur de dents.

Cette bonne image est d'autant plus forte que les patients ont un revenu inférieur à 4000 dirhams, et chez ceux qui gagnent mensuellement un salaire entre 8000-30 000 dirhams.
Les patients dont le revenu est inférieur à 4000 dirhams, considèrent le médecin dentiste comme un soignant (75\%) et professionnel (14\%), alors que les patients avec un revenu entre

8000-30 000 dirhams, perçoivent le médecin dentiste comme un professionnel (30\%), soignant (54\%) et/ ou un artiste (11\%) (Tableau II).

Cette bonne image est aussi corrélée au motif de consultation esthétique, et chez les patients qui consultent par motif de douleur.

Chez les patients qui consultent pour souci esthétique, le médecin dentiste est un soignant

(64\%), professionnel (25\%), avec une touche artistique. Alors que les patients qui consultent pour douleur, ils ont tendance à considérer le médecin dentiste comme un soignant $(75 \%)$ plus qu'un professionnel (13\%) du moment qu' il soulage leurs douleurs (Tableau III).

Le revenu mensuel et les motifs de consultation constituent des facteurs déterminants pour la perception du médecin dentiste. Puisque le revenu mensuel est le critère majeur pour pouvoir accéder aux soins

\begin{tabular}{|c|c|c|c|c|}
\hline Variable (\%) & $<4000 \mathrm{Dh}$ & 4000-8000 Dh & 8000-30000 Dh & $>30000 \mathrm{Dh}$ \\
\hline \multicolumn{4}{|l|}{ Revenu mensuel du ménage } & $\mathrm{P}=0,01$ \\
\hline Soignant & 75 & 73 & 54 & 100 \\
\hline Artiste & 1 & 2 & 2 & 0 \\
\hline Arracheur de dents & 9 & 7 & 4 & 0 \\
\hline Professionnel de santé & 14 & 13 & 3 & 0 \\
\hline Soignant + Artiste & 0 & 5 & 9 & 0 \\
\hline Professionnel + Bonne réputation & 1 & 0 & 0 & 0 \\
\hline
\end{tabular}


$\checkmark$ Tableau III : Perception du médecin dentiste en fonction des motifs de consultation.

\begin{tabular}{|c|c|c|c|c|c|c|}
\hline Variable (\%) & 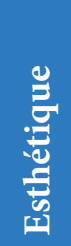 & 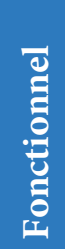 & 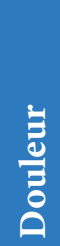 & 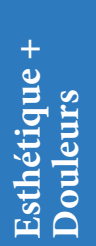 & 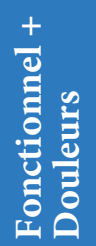 & 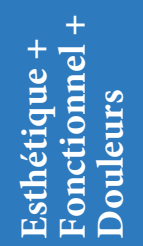 \\
\hline Motifs de consultation & & & & & & $\mathrm{P}=0,000$ \\
\hline Soignant & 64 & 61 & 75 & 82 & 73 & 58 \\
\hline Artiste & 2 & 3 & 2 & 0 & 0 & 0 \\
\hline Arracheur de dents & 0 & 11 & 9 & 9 & 0 & 0 \\
\hline Professionnel de santé & 25 & 21 & 13 & 9 & 13 & 42 \\
\hline Soignant + Artiste & 9 & 5 & 0 & 0 & 7 & 0 \\
\hline Professionnel + Bonne réputation & 0 & 0 & 2 & 0 & 0 & 0 \\
\hline
\end{tabular}

bucco-dentaires, alors que le motif de consultation constitue le point de départ de la relation dentiste-patient : cette première impression sur laquelle se bâtit toute la relation.

\section{PERCEPTION DU «BON » MÉDECIN DENTISTE}

Dans les études précédentes, l'effet de caractéristiques sociodémographiques des patients sur la perception du «bon » médecin dentiste n'était pas évident.

Dans cette enquête aussi, aucune différence significative n'a été observée pour le comportement, la communication avec le médecin dentiste, et les compétences cliniques.

En outre, pour tous les patients marocains un «bon » médecin dentiste est celui qui : se comporte bien avec les patients, maîtrise les compétences techniques, et arrive à communiquer avec ses patients.

Mais on retrouve des différences significatives pour les paramètres suivantes.

\section{Présentation et situation du cabinet}

À Strasbourg, l'étude menée par Devaux M. (1985) [3] a révélé que seulement 17,24 \% tiennent compte de la salle d'attente, où la lecture est la plus importante, et que les plus à être influencés par cette salle ceux de niveaux social moins défavorisé.

Et dans l'étude menée à Strasbourg (1985) (3), la salle de soins a un peu plus d'importance lors du choix du praticien, puisque $47,69 \%$ de la population déclarent tenir compte de ce critère et préfèrent plus l'équipement moderne qu'un aménagement soigné. Et $50 \%$ des patients prennent en considération la situation $\mathrm{du}$ cabinet, et ils préfèrent aussi la proximité par rapport au domicile.

Par contre l'importance donnée à ce critère est plus évidente d'après l'étude menée par Arora R. (1999) [4] dans l'état du Missouri auprès de 144 sujets adultes. Notre enquête a révélé que pour $6,5 \%$ des patients, le «bon» médecin dentiste est celui qui a cabinet dentaire bien présenté, ça regroupe en même temps l'aménagement confortable, l'équipement moderne, et être situé le plus proche possible du lieu de résidence avec un accès facile comme parking, ascenseur...

Dans notre enquête, cette attitude dépend de l'âge, $\mathrm{du}$ type de profession, du niveau d'instruction, du revenu mensuel, et du motif de la consultation : elle est marquée plus chez les personnes âgées de 60 ans et plus (27\%), chez ceux dont le revenu mensuel est supérieur à $30000 \mathrm{DH}(100 \%)$ et entre 8000-30000 DH (10\%), chez ceux de niveau primaire (19\%) et analphabète ( $17 \%$ ), chez les retraités (43\%) et ceux dont le motif de consultation était pour des soins esthétiques + fonctionnelles (25\%) (Tableaux IV, V, VI, VII).

\section{Compétence et méthode de travail}

Dans l'article 24 du code de déontologie : le médecin dentiste qui a accepté de donner des soins à un malade doit lui assurer des soins éclairés et conformes aux données acquises de la science [5].

Le médecin dentiste taille, lime, perfore et fait saigner une zone particulièrement investie dans une relation de face-à-face, où l'angoisse, mais aussi l'insatisfaction et l'agressivité de l'opéré peuvent toujours se manifester, et le dentiste est immédiatement confronté au résultat de son travail [6].

Cependant, les critères objectifs, fonctionnels, prophylactiques ou techniques sont presque toujours 
$\checkmark$ Tableau IV : Perception du « bon » médecin dentiste en fonction du type de la profession.

\begin{tabular}{|l|c|c|c|c|c|c|}
\hline \multicolumn{1}{|c|}{ Variable (\%) } & $\begin{array}{c}\text { Activité } \\
\text { régéluière }\end{array}$ & Retraité & Sans & $\begin{array}{c}\text { Étu- } \\
\text { diants }\end{array}$ & Autres \\
\hline Compétences & 71 & 57 & 64 & 71 & 67 & 0,86 \\
\hline Comportement & 80 & 57 & 64 & 67 & 54,5 & 0,05 \\
\hline Communication & 70 & 71 & 57 & 69 & 51,5 & 0,33 \\
\hline Honoraires & 17 & 14 & 39 & 6 & 48,5 & 0,000 \\
\hline Facteurs personnels & 4 & 0 & 14 & 6 & 9 & 0,38 \\
\hline Présentation et situation du cabinet & 8 & 43 & 11 & 0 & 3 & 0,003 \\
\hline Hygiène et stérilisation & 18 & 0 & 14 & 20 & 9 & 0,55 \\
\hline Autres & 10,5 & 14 & 4 & 4 & 9 & 0,52 \\
\hline
\end{tabular}

- Tableau V : Perception du « bon » médecin dentiste en fonction du niveau d'instruction.

\begin{tabular}{|l|c|c|c|c|c|}
\hline \multicolumn{1}{|c|}{ Variable (\%) } & Analphabète & Primaire & Secondaire & Universitaire & P \\
\hline Compétences & 50 & 81 & 47 & 96 & 0,33 \\
\hline Comportement & 67 & 75 & 56 & 72 & 0,29 \\
\hline Communication & 58 & 62,5 & 50 & 69 & 0,21 \\
\hline Honoraires & 42 & 62,5 & 32 & 15 & 0,000 \\
\hline Facteurs personnels & 33 & 6 & 9 & 4 & 0,05 \\
\hline Présentation et situation du cabinet & 17 & 19 & 0 & 6 & 0,02 \\
\hline Hygiène et stérilisation & 0 & 25 & 9 & 18 & 0,17 \\
\hline Autres & 8 & 6 & 12 & 7 & 0,75 \\
\hline
\end{tabular}

incompréhensibles pour le patient, et seuls restent pour guider son choix, les critères esthétiques et financiers sinon des critères subjectifs tels que les méthodes de travail (ça ne fasse pas mal, rapide, bien organisé, etc.) [6-8].

En ayant recours au médecin dentiste, le patient cherche avant tout à annuler cet état de souffrance et il est conscient de l'évolution technologique qu'a connue l'odontologie, ce patient a retenu que l'on souffrait moins chez le médecin dentiste car il y a l'anesthésie, matériel rotatif, laser... [9].

Une étude menée aux États-Unis en 1999 [4] par Arora R. dans l'état du Missouri a révélé que les soins sans douleurs étaient un critère très important lors du choix du médecin dentiste.

En Angleterre, une étude réalisée en 1995 [10] a révélé que les critères de choix les plus importants sont en rapport avec les méthodes de travail, et vient en premier lieu : un dentiste attentionné et avenant puis les soins sans douleurs.

Notre enquête a révélé que pour désigner le "bon » médecin dentiste, les patients accordent beaucoup d'importance à la méthode du travail puisque $68,8 \%$ les citent comme $2^{\text {e }}$ critère majeur pour être un «bon " médecin dentiste et exigent surtout des soins sans douleurs, arrivent ensuite l'habilité, les techniques récentes, l'explication des procédures et la rapidité dans l'exécution des soins.

Cette attitude est corrélée à l'âge, elle est plus marquée chez les patients âgés de 18 à 39 ans (86 \%) et de 40 à 59 ans $(73 \%)$.

L'exigence des patients pour des soins exempts de douleurs parait évidente du moment que la douleur constitue le motif de consultation le plus fréquent.

\section{Les honoraires}

L'exercice libéral suppose que le médecin dentiste perçoive directement de la part du patient sous la forme d'honoraires la rémunération de son travail. Le praticien reçoit des honoraires pour le temps et les connaissances qu'il met à la disposition des patients [11].

Le praticien applique un tarif personnel qu'il a lui-même déterminé en fonction de paramètres qui lui sont propres dont il est seul juge : titres, compétences, lieu d'exercice, standing, nature de la clientèle, etc.

Aussi dans le cas où le patient peut choisir son praticien, il possède du même coup la liberté de le quitter en n'acceptant pas le montant des honoraires [6]. 
$\checkmark$ Tableau VI : Perception du «bon »médecin dentiste en fonction du revenu mensuel du ménage.

\begin{tabular}{|c|c|c|c|c|c|}
\hline Variable (\%) & $<4000 \mathrm{Dh}$ & 4000-8000 Dh & 8000-30000 Dh & $>30000 \mathrm{Dh}$ & $\mathbf{P}$ \\
\hline Compétences & 72 & 63 & 69 & 0 & 0,44 \\
\hline Comportement & 62 & 68 & 82 & 100 & 0,08 \\
\hline Communication & 60,5 & 61 & 73,5 & 0 & 0,20 \\
\hline Honoraires & 30 & 29 & 8 & 0 & 0,009 \\
\hline Facteurs personnels & 8 & 8 & 2 & 100 & 0,04 \\
\hline Présentation et situation du cabinet & 3 & 6,5 & 10 & 100 & 0,02 \\
\hline Hygiène et stérilisation & 10,5 & 19 & 18 & 100 & 0,08 \\
\hline Autres & 7 & 8 & 8 & 0 & 0,86 \\
\hline
\end{tabular}

$\checkmark$ Tableau VII : Perception du «bon » médecin dentiste en fonction des motifs de consultation.

\begin{tabular}{|c|c|c|c|c|c|c|c|c|}
\hline Variable (\%) & 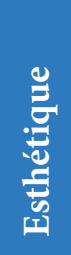 & 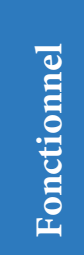 & $\frac{\overrightarrow{0}}{\frac{0}{0}}$ & 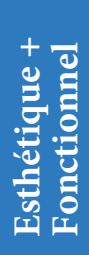 & 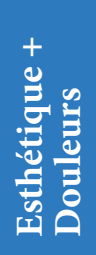 & 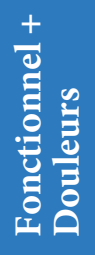 & 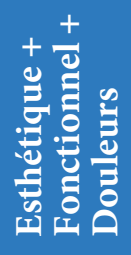 & $\mathbf{P}$ \\
\hline Compétences & 66 & 58,5 & 75 & 75 & 73 & 73 & 67 & 0,71 \\
\hline Comportement & 70 & 58,5 & 65 & 100 & 91 & 80 & 83 & 0,22 \\
\hline Communication & 62 & 56 & 62 & 75 & 82 & 67 & 92 & 0,30 \\
\hline Honoraires & 15 & 22 & 25 & 0 & 27 & 53 & 25 & 0,12 \\
\hline Facteurs personnels & 6 & 7 & 6 & 0 & 27 & 0 & 0 & 0,27 \\
\hline Présentation et situation du cabinet & 2 & 10 & 3 & 25 & 18 & 7 & 17 & 0,04 \\
\hline Hygiène et stérilisation & 19 & 12 & 6 & 25 & 36 & 33 & 33 & 0,005 \\
\hline Autres & 6 & 12 & 9 & 0 & 9 & 0 & 0 & 0,75 \\
\hline
\end{tabular}

D'après l'étude menée par ABID H. en 2002 [12], les problèmes d'argent représentent $45,5 \%$ des freins à la consultation chez le dentiste.

L'étude menée dans l'état d'Ohio en 1980 [13] a révélée que seulement $39 \%$ des personnes questionnées choisissent leur dentiste car il pratique des prix raisonnable et seulement $7 \%$ le considèrent comme étant le critère le plus important.

Concernant les modes de paiement, il apparaît que le règlement des honoraires sur plusieurs tranches soit le moyen le plus adapté.

Les résultats de notre enquête confirment l'importance que prennent les honoraires pratiqués ainsi que les modes de paiement dans la perception du «bon » médecin dentiste, puisque $23,5 \%$ des patients en tiennent compte, alors qu'à Strasbourg (1985) [3] $30,5 \%$ des patients les prennent en considération.

Pour nos patients marocains, cette attitude est liée au lieu de résidence rural par $47 \%$, au niveau d'étude primaire $(62,5 \%)$ et analphabète $(42 \%)$, elle est marquée chez les individus dont le revenu mensuel est inférieur à 4000 dirhams (30\%) et compris entre
4000-8000 Dirhams (29\%), et chez ceux qui sont sans activité $(39 \%)$ ou avec des activités non régulières (48,5 \%) (Tableaux IV, V, VII).

\section{Facteurs liés à la personne du dentiste}

L'image sociale du dentiste était peu valorisante, personnage dangereux (parce qu'il fait mal et qu'il coûte cher), cependant le dentiste sadique, puissant et craint semble néanmoins avoir disparu.

Actuellement, il est à la fois le personnage rassurant, protecteur et sympathique qui restaure la fonction et l'esthétique de la bouche, revalorise l'image de soi aussi bien personnelle que relationnelle, il est aussi le personnage puissant, doté de pouvoirs, de droits et de diplômes, dont on reconnaît une grande compétence technique [14].

La relation qui s'établie accentue plus cette inégalité, elle met le patient en situation d'infériorité, d'une part par la position qu'il occupe sur le fauteuil et d'autre part par le lieu même de l'intervention : la bouche, organe d'une grande importance psychologique et affective [65]. 
Pour toutes ces raisons, le patient choisira son «bon » praticien, celui qui correspondra le mieux à l'image qu'il s'en fait.

Notre enquête a révélée qu'à peu près 6,5\% des patients tiennent compte de la personne du dentiste pour juger le «bon » dentiste, et généralement le sexe (féminin) du médecin dentiste est le critère le pus important pour ces patients.

Cette attitude est liée au niveau d'étude chez les patients analphabète $(33 \%)$, et remarquée pour un patient dont le revenu mensuel est supérieur à 30000 dirhams où son «bon " médecin dentiste est celui qui est âgée (Tableaux V, VI).

\section{L'hygiène et stérilisation}

Notons simplement que depuis quelques années, les patients sont attentifs aux conditions d'hygiène, de stérilité et de non contamination, dans notre exercice. Dans une étude menée par "Clinic actu » 2007 [17] dont la question était : sur quoi repose la confiance que vous accordent vos patients ? Les résultats étaient comme suit : l'équipement et la propreté du cabinet apparaissent primordiaux pour $8 \%$ des patients consultés pour avoir confiance en leur médecin dentiste, après la relation humaine par $68 \%$ et la démarche médicale $20 \%$.

Et dans une étude menée en Grèce 1999 [18], pour déceler les attentes et les perceptions de patients grecs concernant la qualité de santé publique dentaire, les patients ont mentionné les règles de l'antisepsie et la stérilisation comme priorité majeur de leur attente et exigence en soins dentaires.

L'hygiène et l'asepsie est le critère le plus exigé dans la salle de soins, même si seulement $16 \%$ de notre échantillon en tiennent compte.

Dans notre enquête, cette attitude est liée au motif de consultation, elle est remarquée chez les patients consultants pour des soins esthétiques et fonctionnels ou associées. Et seulement $6 \%$ des patients parmi ceux qui consultaient pour douleurs dentaires : ces patients consultaient en urgence et n'avait peut être pas le temps de se soucier de la stérilisation et l'hygiène du cabinet, ils voulaient seulement se soulager de leur souffrance. Pour ces patients, la santé est leur dernier souci puisqu'ils attendent la survenu de la douleur pour consulter, ce manque de souci pour leur bien-être peut-il expliquer le manque d'attention pour la stérilisation et l'hygiène du cabinet (Tableau VII).

\section{Autres facteurs}

La définition de certains patients pour le "bon» médecin dentiste ne figurait pas dans les différentes catégories déjà cités, mais ils donnaient d'autres définitions $(7.5 \%)$ comme :

$\checkmark$ le « bon » médecin dentiste n'existe pas. parle peu.

I celui qui s'adapte à mes horaires de travail.

I son travail doit être accompli dans trois séances au maximum.

Ijoignable en weekend.

Ce sont plutôt des remarques, des idées, ou des suggestions données par ces patients. Ils ont un intérêt majeur pour le développement de la relation avec le médecin dentiste.

Pour BINHAS E : le patient qui exprime son mécontentement nous rend un immense service.

Il n'y a rien de pire qu'un patient insatisfait qui se tait mais... qui ne revient pas. Laissez vos patients s'exprimer, c'est créer une situation positive qui permet de renforcer la relation de confiance établie entre eux et tous les membres du cabinet.

Votre capacité à répondre aux insatisfactions d'un patient peut consolider de façon significative votre relation avec lui. Elle peut souvent propulser cette relation vers des niveaux jamais atteints auparavant. À l'évidence, les meilleurs cabinets ont tous en commun un haut niveau de service, d'écoute et d'empathie [19].

Dans notre enquête, ces définitions du «bon » médecin dentiste est liée au sexe, et à la consultation antérieure. Elle est plus marquée chez les hommes (13\%), et chez les patients qui n'ont jamais consulté un médecin dentiste (20\%).

\section{CONCLUSION}

À l'issue de notre enquête, il apparaît que les personnes interrogées font une différence entre le médecin dentiste et le «bon " médecin dentiste.

Elles utilisent davantage de termes pour qualifier le bon médecin dentiste et les types de la compétence, sont également différents, recourant plus souvent à des mots qualifiant des dimensions relationnelles, affectives et morales pour le « bon » médecin dentiste. La qualité de la relation humaine (comportement du médecin dentiste avec le patient et sa communication avec lui) est le critère le plus recherché par les patients. Ce premier critère est suivi de la qualité du travail, les patients exigent surtout des soins exempts de douleurs. Les honoraires occupent une place importante lors de la perception. En effet, le facteur économique constitue un obstacle à l'accès aux soins dentaires en raison du pouvoir d'achat limité du citoyen marocain.

Enfin la stérilisation et l'hygiène du cabinet sont parmi les facteurs déterminants le «bon » médecin dentiste. Toute l'intention des médecins dentistes devrait donc porter sur l'impression laissée aux patients tant sur le plan technique que relationnel. 
En effet, les connaissances techniques ne constituent plus le seul facteur décisif de la réussite du traitement dentaire, celle-ci dépend aussi de la prise en compte des dimensions humaines et sociales. Le médecin dentiste doit savoir qu'il ne soigne pas une dent, ni des dents, ni même une bouche, mais qu'il soigne un malade qui lui a confié sa bouche.

Cette prise de conscience devrait conduire le praticien à s'informer voir se donner une formation en communication afin de dépasser le rôle de technicien de l'art dentaire et d'assumer son rôle de médecin dentiste traitant son patient dans sa dimension humaine.

Les résultats de cette étude mériteraient peut-être d'être intégrés à un ensemble d'informations transmises aux étudiants qui envisagent d'entreprendre des études de médecine dentaire. Les modifications du comportement professionnel des étudiants au cours de leur curriculum sont un paramètre mal connu. L'attente des patients à leur égard est donc une information à leur transmettre tôt. Une enquête sur ce qu'ils pensent que les patients attendent d'eux serait intéressante.

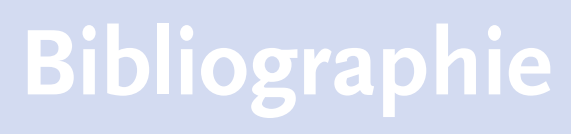

[1] 2- Binhas E. Perception = réalité. Clinic, mai $2007,28: 16$.

[2] 50- L'association dentaire française. Les français et leur chirurgien-dentiste. L'information dentaire, 5 décembre 2007, $42: 2725$.

[3] 63- Devaux M. Les facteurs susceptibles d'influencer le libre choix du praticien ; d'après une enquête effectué auprès des patients adultes de Strasbourg. Thèse chirurgie dent. Strasbourg $1985 \mathrm{n}^{\circ} 44$.

[4] 64- Arora R. Influence of pain-free dentistry and convenience of dental office on the choice of a dental practitioner: an experimental investigation. Health marketing quarterly 1999 , $16(3): 43-54$.

[5] 21- Code de déontologie des médecins dentistes du Maroc. Décret n ${ }^{\circ}$ 2-96-989 du
5 janvier 1999. Publié au bulletin officiel $n^{\circ} 4662$ du 17 chaoual 1419 (4 février 1999).

[6] 22- Hugly C. L'argent dans la relation thérapeutique. Revue d'odontologie 1981, 10 (3) : 239-244.

[7] 23- Hescot P. Les satisfactions et attentes des patients à l'égard de leur chirurgiendentiste. Collection de l'ADF 1996.

[8] 24- Benchekroun S. Chirurgiens-dentistes : l'alarme. Espérance médicale, spéciale dentaire, février 2001, 8 (20) : 3-8.

[9] 25- Belair J.P. La demande du patient en odontologie. Revue d'odontologie 1981, 10 (3) : 219-221.

[10] 65- Holt V.P., MC Hugh K. Factors influencing patient loyalty to dentist and dental practice. British dental journal, November 1997, 183 (10): 365-370.

[11] 36- Charon J. Service patient service gagnant. Édition CDP, Vélizy 1996.

[12] 52- Abid H. L'accès aux soins buccodentaires au Maroc. Thèse : Médecine Dentaire, Rabat, 2002, 50/02.
[13] 66- Garfunkel E. The consumer speaks: how patients select and how much they know about dental health care personnel. The journal of prosthodontic dentistry, April 1980, 43 (4): 380-384

[14] 10- Thery-Hugly MC, Todorova. Relation praticien-patient. EMC odontologie 1998, 23840-C-10.

[15] 67- Battegay R. Psychologie en médecine dentaire « signification psychologique des dents et des interventions sur celles-ci ». Revue mensuelle suisse odontostomatologie, 1994, 104 (8) : 977-980.

[16] 60- Enquête Vos patients vous font-ils confiance ? Clinic, février 2007, 28 : 12-15.

[17] 68- Karydis A., Komboli K.M., Hatzigeorgiou D., Pannis V. Expectations and perceptions of Greek patients regarding the quality of dental healthcare. International journal for quality in health care, 2001, 13 (5): 409-416.

[18] 44- Binhas E. Laissez vos patients s'exprimer. Clinic, mars 2007, $28: 17$. 